\title{
Obesity and Obese-related Chronic Low-grade Inflammation in Promotion of Colorectal Cancer Development
}

\author{
Lukasz Pietrzyk $^{1,2 *}$, Anna Torres ${ }^{1}$, Ryszard Maciejewski $^{1}$, Kamil Torres $^{1}$
}

\begin{abstract}
Colorectal cancer (CRC) is a worldwide health problem, being the third most commonly detected cancer in males and the second in females. Rising CRC incidence trends are mainly regarded as a part of the rapid 'Westernization' of life-style and are associated with calorically excessive high-fat/low-fibre diet, consumption of refined products, lack of physical activity, and obesity. Most recent epidemiological and clinical investigations have consistently evidenced a significant relationship between obesity-driven inflammation in particular steps of colorectal cancer development, including initiation, promotion, progression, and metastasis. Inflammation in obesity occurs by several mechanisms. Roles of imbalanced metabolism (MetS), distinct immune cells, cytokines, and other immune mediators have been suggested in the inflammatory processes. Critical mechanisms are accounted to proinflammatory cytokines (e.g. IL-1, IL-6, IL-8) and tumor necrosis factor- $\alpha$ (TNF- $\alpha$ ). These molecules are secreted by macrophages and are considered as major agents in the transition between acute and chronic inflammation and inflammation-related CRC. The second factor promoting the CRC development in obese individuals is altered adipokine concentrations (leptin and adiponectin). The role of leptin and adiponectin in cancer cell proliferation, invasion, and metastasis is attributable to the activation of several signal transduction pathways (JAK/STAT, mitogen-activated protein kinase (MAPK), phosphatidylinositol 3 kinase (PI3K), mTOR, and 5'AMPK signaling pathways) and multiple dysregulation (COX-2 downregulation, mRNA expression).
\end{abstract}

Keywords: Obesity - colorectal cancer - inflammation - body mass index - interleukin - adiponectin - leptin

Asian Pac J Cancer Prev, 16 (10), 4161-4168

\section{Introduction}

Colorectal cancer (CRC) is a worldwide health problem, the third most commonly detected cancer in males and the second in females. Incidence and mortality rates from related deaths have been on the rise worldwide, e.g. in Europe, the occurance has consistently been increasing over the past two or three decades. In 2012, there were nearly 1.4 milion newly diagnosed cases of CRC with prognosis of 2.4 milion cases of CRC to be diagnosed annually worldwide by 2035. Data from 2012 show a varied CRC incidence across the world with higher values in more developed countries $(737,000$ cases $)$ in comprison to less developed regions (624,000 cases). Based on the 2009-2011 data, the estimated lifetime risk of developing CRC is about 1 in $20(4.7 \%)$ and it is slightly lower in women than in men (American Cancer Society, 2014). In 2012, worldwide mortality due to CRC was 694,000 and contributed to nearly $9 \%$ of all deaths caused by cancers (Ferlay et al., 2013).

Several factors such as genetics, gender, ethnic origin, geographical region, and environmental conditions are attributable to the CRC risk (Haggar and Boushey, 2009).
When taking into account the statistical data, rising CRC incidence trends are mainly regarded as a part of the rapid 'westernization' of the life-style (Winkels et al., 2014). Increasing epidemiological evidence has demonstrated that a raised incidence of CRC is associated with calorically excessive high-fat/low-fibre diet, consumption of refined products, lack of physical activity, and obesity (Birmingham et al., 2009; Morrison et al., 2013).

Furthermore, overweight and obesity has doubled worldwide since 1980 and the epidemic is still rising dramatically (Winkels et al., 2014). Previous reviews by Frezza et al. (2006) suggested that obesity was associated with a 7\%-60\% greater risk of CRC compared with normal-weight people, while updated reviews report an increase to 30\%-70\% greater risk (Bardou et al., 2013).

Reducing the rates of CRC will require both coordination of public health programs and a better understanding of the CRC etiology. The exceeded body weight is a potentially modifiable factor, therefore the understanding of associations between overweight or obesity and CRC is a major challenge, when determining an appropriate strategy for primary prevention of colorectal cancer. Moreover, a better understanding of the 
obesity-driven CRC etiology might help in risk evaluation, diagnosis, and treatment of CRC.

Critical questions include the mechanisms by which metabolism disorders induce inflammation in obesity, and how this inflammatory process initiates the development and progresion of CRC in particular. Recent studies try to explain the role of distinct immune cells, cytokines, and other immune mediators in the particular steps of colorectal carcinogenesis, including initiation, promotion, progression, and metastasis.

Therefore, in this rewiev, we summarize the significance and association between obesity and obeserelated adipokines and inflammatory pathways involved in initiation of colorectal carcinogenesis, promotion and progression. To provide a systematic review for addressing the association between obesity, adipokines, and CRC, a PubMed search was conducted using keywords related to colorectal cancer and obesity, obese-related adipokines (leptin, adiponektin, proinflammatory cytokines), and adipose tissue infiltrating cells (macrophages and neutrophils). The PubMed search was limited to studies published in English between January 2000 and December 2014 due to the substantial progress in investigation of colorectal carcinogenesis, progression, and metastasis. Articles were investigated for relevance by the authors (ŁP and KT) and 89 articles were finally included.

\section{Obesity Markers and Relation to CRC Development}

Excess body weight is defined as abnormal or excessive fat accumulation in adipose tissue that may impair health and increase mortality. The classification of excess body weight is based on the body mass index (BMI), according to which overweight is defined as BMI $>25-29.9 \mathrm{~kg} / \mathrm{m} 2$, and obesity as BMI $>30 \mathrm{~kg} / \mathrm{m} 2$. The disadvantage of the BMI is that it does not identify the distribution of the body fat. The body fat is deposited mainly in visceral and subcutaneous compartments. Abnormal fat accumulation and distribution are gender specific; females are characterized by a greater amount of peripherally located subcutaneous fat, while men have more fat located in the central visceral compartment. Individual variations, age-related differences, body composition, or ethnic factors have to be considered in the diagnosis of overweight or obesity. For example, a relatively high BMI is associated with muscular patients, who have a low visceral fat deposit, on the contrary, some people classified as normal weight based on the BMI may exhibit high fat deposits (Tchernof and Després, 2013). Therefore, the role of the BMI in the prediction of developing colorectal cancer (CRC) is uncertain and should be interpreted together with multiple other BMIrelated factors (body fat composition and distribution, diet, life-style, life-quality) (Winkels et al., 2014).

Current recommendations suggest measuring the waist (WC) and hip circumferences (HC) or calculating the waist-to-hip ratio (WHR) as the most accurate and precise indicators of fat accumulation and visceral obesity. WC equal to or higher than $88 \mathrm{~cm}$ (in women) or $94 \mathrm{~cm}$ (in men) and WHR above 0.90 for males and 0.85 for females are cut off points associated with a high metabolic risk (Nishida et al., 2010). A recent meta-analysis of 21 studies has revealed that a $10-\mathrm{cm}$ increase in WC is related to an increased risk of colorectal adenoma (CRA), regardless of the geographic region, gender, and confounders: alcohol use, smoking status, and family history of colorectal cancer (Hong et al., 2012; Kaneko et al., 2014).

Adipose tissue is composed of various cell types including pre-adipocytes, adipocytes, macrophages, lymphocytes, and fibroblasts and thus it forms an active metabolic and endocrine tissue which functionis altered in obesity (Tchernof et al., 2013; Saely et al., 2012; Coelho et al., 2013). Particularly, obesity with central visceral fat accumulation (VAT) has been associated with a higher risk of the metabolic syndrome (MetS) and may enhance the endocrine avtivity of the tissue (Nishida et al., 2010). In fact, the European Prospective Investigation into Cancer and Nutrition (EPIC) reported that visceral obesity (VAT), rather than the total amount of fat, is linked to a strong risk factor for CRC regardless of the gender, age, or ethnic background (Pischon et al., 2006). Similarly, an association of VAT with CRA was evidenced in the Asian population (Otake et al., 2005; Kang et al., 2010). However, there are reports where the association of VAT with a risk for CRC is questionable. For example, the VAT area did not differ between CRC patients and control patients in the Turkish population (Erarslan et al., 2009); however, the small cohort (only 54 patients) was the study limitation. Recently, the impact of VAT on the normaladenoma sequence but not the adenoma-early carcinoma sequence has been evidenced (Choe et al., 2013). Carcinogenesis is a multistage process of transformation of normal cells into cancer cells and the "adenomacarcinoma sequence" is under control of multiple factors (Li et al., 2014). Therefore, the role of VAT in developing CRC should be interpreted at each step of this sequence, especially as different molecular/metabolic/inflammatory syndromes are recognized as carcinogens in obesity (Choe et al., 2013). However, it has been postulated recently that injuries of colorectal mucosa tissues play a basic role in carcinogenesis initiation. Several factors may invoke the injury-mechanical and chemical, biological infections, as well as chronic inflammation (Erarslan et al., 2009; Mariani et al., 2014).

\section{Obesity-driven Inflammation and CRC}

Most recent epidemiological and clinical investigations have consistently evidenced a significant relationship between obesity-driven inflammation in particular steps of colorectal cancer development, including initiation, promotion, progression, and metastasis (Terzic et al., 2010).

A model of the relationship between obesity and CRC is presented in Figure 1.

The link between the long-standing low-grade inflammation and the pathogenesis of CRC has been proven by many clinical reports, which documented that patients with chronic inflammatory bowel disease have a higher risk of CRC compared to healthy individuals 


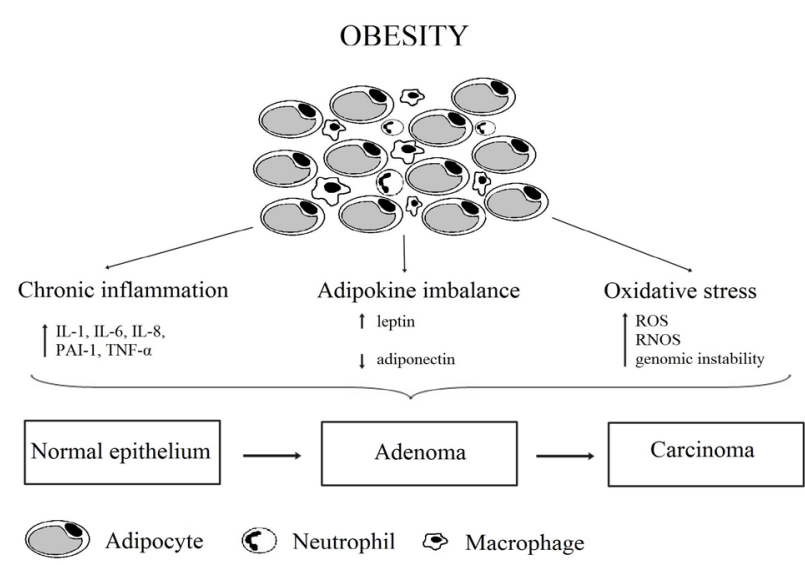

Figure 1. Conceptual Model for the Relationship between Obesity and CRC initiation and development. Adipose tissue considered as a metabolic tissue infiltrated by inflammatory cells macrophages and neutrophils. These cells produce adipocytokines, which favor tumor proliferation and angiogenesis. IL - interleukin, PAI - plasminogen activator inhibitor, TNF - tumor necrosis factor, ROS - reactive oxygen species, RNOS - reactive nitrogen species

(Lukas, 2010; Mattar et al., 2011; Vendramini-Costa and Carvalho, 2012). On the contrary, therapies with the use of anti-inflammatory drugs (e.g. aspirin) significantly reduce the CRC risk (Cole et al., 2009). A recent report from Chinese male population (288 CRC patients vs. 576 control individuals) revealed a positive relationship between biomarkers of chronic inflammation (C-reactive protein) and CRC (Aleksandrova et al., 2010; Wu et al., 2013). A former review of Tsilidis et al. (2008) also related the $\mathrm{CRC}$ risk to the level of circulating $\mathrm{C}$-reactive protein, however mainly in males.

Inflammation in obesity occurs by several mechanisms. The role of imbalanced metabolism (MetS) distinct immune cells, cytokines, and other immune mediators are suggested in the development of the inflammatory process (Esposito et al., 2012; Kabat et al., 2012).

First, the accumulation of macrophages and soluble mediators (adipokines) secreted mainly by adipocytes is a critical stress factor in the development of obesityinduced inflammation (Hotamisligil, 2006; Procaccini et al., 2013). Indeed, parallels have been drawn between excess body weight and secretion of inflammation-related adipokines such as interleukin-6 (IL-6), plasminogen activator inhibitor-1 (PAI-1), and C-reactive protein (CRP) (Tsilidis et al., 2008). These pro-inflammatory adipokines are believed to have direct pro-carcinogenic properties in the gastrointestinal tract (Roberts et al., 2010; Landskron et al., 2014; Mariani et al., 2014).

Macrophages play an important role in both tumor initiation and various key steps in its growth and metastasis (Mosser and Edwards, 2008). Additionally, two types of macrophages have been identified as the primary source of many circulating inflammatory molecules that are detected in the obese state (Gregor and Hotamisligil, 2011). Type I (M1) macrophages secrete a wide array of powerful chemical substances, e.g. enzymes, proinflammatory cytokines, regulatory factors, interleukin-12 (IL-12), and are also involved in termination of tumor cells by secreting tumor necrosis factor- $\alpha$ (TNF- $\alpha$ ), reactive oxygen species (ROS), and nitrogen species (RNOS). On the contrary, type II macrophages (M2) moderate the inflammatory response, eliminate cell wastes, promote angiogenesis and tissue remodeling, and release cytokines, including anti-inflammatory interleukin-10 (IL-10) (Mariani and Edwards, 2008; Gordon, 2003; Franklin et al., 2014).

The number of macrophages with the M1 phenotype increases rapidly during chronic inflammation and it may result in predestination of a given tissue to tumor initiation by the release of factors that promote neoplastic transformation. The tumor-associated macrophages (TAMs) express the M2 phenotype, promote tumor cell survival, and contribute to tumor growth, vascularization, and progression (Gordon, 2003; Talmadge et al, 2007). Macrophages have been shown to infiltrate a CRC tumor (Solinas et al., 2009). High density of TAMs has been significantly associated with poor prognosis in a majority of tumors (Balkwill et al., 2005; Erreni et al., 2011); however, the invasiveness of CRC cells and their ability to metastasize to other organs is associated with the ratio of M2 to M1 but not the number of M2 phenotype cells (Serhan, 2007; Cui et al., 2013).

The second type of inflammatory cells involved in pathogenesis of CRC is neutrophils (Erreni et al., 2011). In colorectal carcinogenesis, proinflammatory cytokines (e.g. IL-1, IL-6, IL-8) overproduced by macrophages are rapidly recruited to the site of infection in endothelial colon cells (Mariani et al., 2014). Biochemical changes in the endothelium initiate cell death. Phagocytosis of apoptotic cells by macrophages might change the expression of cytokines and increase the infiltration of neutrophil cells in peripheral colon tissue. As a result of inflammatory signals (e.g. overproduction of proinflammatory cytokines), neutrophils strengthen the inflammation through the reactive oxygen species (ROS). If the inflammatory status is prolonged, the epithelial cells express mediators (e.g. lipoxins). These lipids block neutrophil migration and stimulate the phagocytosis of apoptotic neutrophils by macrophages M1 (Serhan, 2007). The lack of neutrophils induces the activation of macrophages M2, which secrete anti-inflammatory cytokines (e.g. IL-10). In addition, the secretion of the transforming growth factor-beta (TGF- $\beta$ ) is stimulated and the pathogenesis in the epithelial cells is strengthened. These multiple factors are involved in uncontrolled development of epithelial cells (Cui et al., 2013; Mariani et al., 2014).

\section{Proinflammatory Cytokines}

Another critical mechanism that mediates inflammation in obesity is derivative of proinflammatory cytokines (e.g. IL-1, IL-6, IL-8) and tumor necrosis factor- $\alpha$ (TNF- $\alpha$ ). These molecules are secreted by macrophages and are considered as major agents in the transition between acute and chronic inflammation and inflammation-related CRC (Roberts et al., 2010; Gordon, 2011; Landskron et al., 2014).

Particularly, the involvement of IL-6 in colorectal cancer has been documented (Landskron et al., 2014; Mariani et al., 2014). In vitro studies indicated that IL-6 
encouraged the growth of colon cancer cells, increased the invasiveness of colon cancer cells, and probably promoted secondary tumor formation (Schneider et al., 2000). A clinical study unequivocally evidenced a higher level of IL-6 in the serum of colorectal cancer patients compared to healthy individuals; the increasing level of IL-6 was associated with the tumor grade and poorer survival rate (Knupfer and Preiss, 2010). IL-6 is also a relevant angiogenic factor. High levels of IL-6 correlate with the vascular endothelial growth factor (VEGF) content in colorectal cancer (Kim et al., 2003; Eldesoky et al., 2011; Landskron et al., 2014).

Besides IL-6, several other interleukins have a pro-inflammatory effect. A number of in vivo studies have documented that interleukin-1 (IL-1) produced by macrophages and epithelial cells exerts pro-oncogenic effects in colorectal neoplasia (Lewis et al., 2006). Interleukin- 1 is a crucial regulator of the production of IL-8, IL-6, and growth factors such as TNF- $\alpha$, TGF- $\beta$, and VEGF (Apte and Voronov, 2002; Lee et al., 2012). Proliferation of colon cancer cells is stimulated indirectly by production of angiogenic proteins, and contribution of IL-1 in angiogenesis has recently been determined (Voronov et al., 2003).

An important role in colon cancer pathogenesis is also related to the level of interleukin-8 (IL-8). The relationship between increased levels of IL- 8 in the tumor microenvironment and colon cancer growth and metastasis was documented by Lee et al. (2012).

A special role in the pathophysiology of obesitydriven colon carcinogenesis is related to the tumor necrosis factor-alpha (Osório-Costa and Carvalheira, 2013). The increased level of TNF- $\alpha$ was documented in the microenvironment of CRC (Landskron et al., 2014). TNF- $\alpha$ is a pro-inflammatory adipokine produced mainly by activated macrophages, natural killer (NK) cells, and neutrophils, although it can be produced by many other cell types. Therefore, the TNF- $\alpha$ may influence all stages of cancer development (initiation, promotion, and progression). The TNF- $\alpha$ exerts an impact on the development of colon cancer in obese states through direct effects on colon cells and indirectly through action on the tumor-promoting microenvironment. TNF- $\alpha$ involvement in cancer initiation is based on a dramatic increase in reactive oxygen species (ROS) and nitrogen species (RNOS) levels in adjacent cells. Accordingly, this may result in damage to DNA or cause genomic instability that initiates carcinogenesis (Hermsen et al., 2002; Alemán et al., 2014).

It was evidenced that the $\mathrm{CRC}$ tumor $>3 \mathrm{~mm}$ requires efficient blood supply during promotion. TNF- $\alpha$, in addition to other molecules (VEGF, IL-8), can enhance tumor progression through formation of new blood vessels (angiogenesis). It has been shown that the visceral adipose tissue of patients with colon cancer exhibits increased TNF- $\alpha$ and VEGF levels signalizing that obesity-driven inflammation strongly influences colon cancer promotion (Catalán et al., 2011).

TNF- $\alpha$ may also contribute to cancer progression by promoting cell migration and metastasis (Chambers et al., 2002). The TNF- $\alpha$ can induce overexpression of important regulators of epithelial-mesenchymal transition (EMT), resulting in increased movements and capacity to invade basal membranes and allow cancer cells to reach blood vessels or lymphatic vessels (Kalluri and Weinberg, 2009). Interestingly, TNF- $\alpha$ can promote survival of circulating metastatic cells through activation of molecular pathways, which promote the circulation of cancer cells and allow them to avoid immunological attacks (Osório-Costa and Carvalheira, 2013).

The other important factor mediating tumor growth and metastasis is the vascular endothelial growth factor (VEGF). It is hypothesized that overexpression of the VEGF protein is a turning point in the development of colorectal cancers by activating angiogenesis and tumor neovascularization (Martins et al., 2013). It is suggested that VEGF expression should be quantified as important prognostic information in CRC (Bendardaf et al., 2008).

A crucial role in understanding CRC development and progression is attributed to the mechanism by which the transforming growth factor-beta (TGF $\beta$ ) acts. The TGF $\beta$ is a group of multifunctional polypeptides that function through binding to TGF- $\beta$ receptors. These peptides take part in different cellular processes (e.g. cell proliferation, differentiation, apoptosis, migration, metastasis) (Geng et al., 2013; Liu et al, 2011). Recent studies have indicated that TGF- $\beta$ signaling suppresses metastasis in a subset of colon cancer cells in vivo (Simms et al., 2012).

\section{Obesity- Leptin and Adiponectin}

A growing body of evidence indicates that the metabolic syndrome (MetS) and expression of various adipokines (leptin and adiponectin) associated with obesity plays a crucial role in the development of colorectal cancer (Hardwick et al., 2001; Hoda et al., 2007; Endo et al., 2011; Aleksandrova et al., 2012; Healy et al., 2012; Erkasap et al., 2013; Gialamas et al., 2013). In obese people, the elevated insulin level, the rapidly growing secretion of leptin, and decreased adiponectin levels altogether activate several signaling pathways that favor carcinogenesis and are associated with colon cancer development.

Leptin, the most abundant adipokine, is secreted almost exclusively by white adipose tissue and it circulates in blood serum in both free and bounded forms. In obese individuals, the level of leptin in blood serum is higher compared to those with normal body mass; this dependence has been brought to light by Sauter et al. (2004), who reported that leptin expression strongly correlated with the percentage of body fat. In vitro studies clearly demonstrated that abnormal leptin expression in human colon epithelial cells functions as a growth factor. Leptin or its soluble receptor $(\mathrm{ObRb})$ acts at various stages of neoplastic progression and at stages subsequent to tumor initiation in CRC cell lines (Endo et al., 2011; Erkasap et al., 2013). Moreover, Paik et al. (2009) documented that leptin expression increased gradually in the normal-adenoma-adenocarcinoma sequence of CRC development and promoted their proliferation.

However, in view of epidemiological and clinical reports, the role of leptin in the development of colorectal 
cancer (CRC) and adenoma (CRA) is ambiguous. The level of leptin or ObRb has been found to be associated with an increased risk of CRC, although some study revealed a normal level of leptin in the serum of patients with CRC (Aleksandrova et al., 2012; Aloulou et al., 2008). A recent study (with 3,614 CRC vs 1,215 CRA vs 5,220 controls) found a positive association between serum leptin and the CRA risk, but not with the CRC risk (Gialamas et al., 2013). In vivo investigations of Liu et al. (2011) documented that leptin was overexpressed and bound to its receptor in colorectal carcinoma tissues. According to Wiwanitkit (2007), a prominent role in the CRC development is attributable to the interaction between leptin and the leptin receptor $(\mathrm{ObRb})$; in the colon tissues from CRC patients, the Ob-R mRNA gene expression was correlated negatively with the serum leptin concentration, indicating increased sensitivity to leptin receptors.

The relationship between serum leptin concentrations and CRC progression and aggressiveness has also been reported (Tutino et al., 2011). The expression of leptin, the ObRb receptor, and VEGF correlate positively with the grade of tumor differentiation, therefore the authors suggest that binding of leptin to the $\mathrm{ObRb}$ receptor stimulates the proliferation of CRC cells, which results in tumor overgrowth in CRC. The synergistic function of leptin and VEGF may accelerate angiogenesis and promote cancer invasion and metastasis of carcinoma tissues to other sites and tissues in CRC patients (Bendardaf et al., 2008; Geng et al., 2013; Martins et al., 2013; ). Leptin can also upregulate the expression of other angiogenic factors, such as the basic fibroblast growth factor (FGF), interleukin-1 $\beta$ (IL-1 $\beta$ ), and the leukemia inhibitory factor (LIF) in cancer cells, which results in potentiation of the proangiogenic effects of leptin (Lewis et al., 2006; Endo et al., 2011; Gialamas et al., 2013).

However, the potential link between elevated leptin concentrations and CRC progression and invasiveness depends on different interrelated factors (e.g. gender, MetS, genetic background). Stattin et al. (2004) evidenced that an increasing level of leptin stimulates invasiveness of CRC in men, but not in women. Another study associated the aggressiveness of CRC tumor in male patients with high leptin levels and hypertension, raised plasma glucose, triglycerides, and high-density cholesterol levels (Healy et al., 2012). On the contrary, Ho et. al. (2012) found association between the level of leptin and progression of CRC in postmenopausal women and documented that the effect of hyperleptinemia was mediated mostly by insulin. Tamakoshi et al.(2005) reported that invasiveness of CRC in women is associated with a high level of leptin in serum. It is suggested that only very high levels of leptin and other metabolic factors acting simultaneously (levels of C-peptide, HbA1c, and leptin/ObR ratio) activate and increase CRC proliferation (Stocks et al., 2008).

Thus, it can be concluded that the relationship between obesity, the leptin level, and CRC is multifactorial. The role of leptin in cancer cell proliferation, invasion, and metastasis is attributable to the activation of several signal transduction pathways (Saxena et al., 2007). According to the current knowledge, the leptin-signaling pathway in CRC is mainly transduced by JAK/STAT, mitogenactivated protein kinase (MAPK), phosphatidylinositol 3 kinase (PI3K), mTOR, and 5'AMPK signaling pathways (Ognunwobi and Bealses, 2007).

Adiponectin, an adipokine, is almost exclusively secreted by adipose tissue. The level of the hormone decreases in obesity and is inversely correlated with visceral obesity (Ho et al., 2012; Procaccini et al., 2013; Joshi and Lee, 2014). Adiponectin action is mediated by binding to two main receptors: adiponectin receptor 1 (AdipoR1) and receptor 2 (AdipoR2) (Kadowaki and Yamauchi, 2005). Adiponecitn has an impact on colorectal carcinogenesis by indirect and direct mechanisms (Yoneda et al., 2008; Gulcelik et al., 2012). In the indirect pathway, adiponectin acts through mediators such as insulin and proinflammatory factors. Indeed, a recent study suggests that altered adiponectin levels are engaged in energy imbalance and insulin resisance and have a pro-inflamatory effect (Hotamisligil, 2006; Mattar et al., 2011; Aleksandrova et al., 2012; Landskron et al., 2014; Mariani et al, 2014). Direct involvement of adiponectin in carcinogenesis is through action on cancer cells by contribution to cell proliferation and to the process of tissue remodeling (Tae et al., 2014). Low levels of adiponectin induce apoptosis and stimulate the process of cell polliferation by indirect destabilisation of glucose metabolism and insulin resistance (Kadowaki and Yamauchi, 2005; Yoneda et al., 2008). Hypoadiponectinemia may thus indicate a link between overweight and an increased risk of colorectal cancer. In fact, lower levels of adiponectin have been documented in the serum of CRC patients compared with healthy patients (Otake et al., 2005). On the contrary, no statistically significant association between serum adiponectin and a long-term risk of CRC in men was reported (Wei et al., 2005).

It is hypothethised that the conflicting results of the impact of adiponectin on cancer development and progresion may relate to the structure of the polypeptide in plasma. High molecular weight adiponectin is suggested to be more active in glucose metabolism and contribute to diminished insulin sensitivity than low molecular weight adiponectin (Kadowaki and Yamauchi, 2005).

It has recently been documented that the relationship between adiponectin and colorectal carcinogenesis is not simple (Yoneda et al., 2008; Tae et al., 2014). While examining serum and tissues from patients with CRC, the authors documented that the decreased in serum in the normal-adenoma-carcinoma progression. In addition adiponectin concentrations, decreased expression of adiponectin receptors (AdipoRs) in the colonic epithelium, which leads to increased adiponectin expression in the mesenchyme, was recorded. Therefore, they hypothetise that an increased adiponectin concentration initiates multiple disregulations (e.g. COX-2 downregulation, mRNA expression) that may activate carcinogenesis (Tae et al., 2014). It is suggested that measuring the adiponectin levels and expression of adiponectin receptors (AdipoR1, AdipoR2) in tumor might have diagnostic and predictive values (Yoneda et al., 2008). 


\section{Conclusions}

All in all, colorectal cancer, like any other cancer, has a multifactorial etiology, therefore many factors acting together and inducing cancer development and progression have to be taken into account. The rapidly increasing trends in both CRC cases and obese people noted over several decades indicate that lifestyle revision can support medical therapies to successfully treat and suppress CRC development and metastasis. Particularly, dietary habits and lifestyle practices (e.g. activity levels) may lead to reduction in total calories intake, improved insulin sensitivity, and decreased inflammation should be propagated in obesity. Restoration and maintenance of metabolic balance is a basic tool and therapeutic approach in treating obesity-induced complications. Systematic anti-inflammatory activities seems to be critical in prevention of colon carcinogenesis.

\section{Acknowledgements}

This work was supported by the Polish Ministry of Science and Higher Education (grant \# NN403 393039 to R.M.)

\section{References}

Aleksandrova K, Jenab M, Boeing H, et al (2010). Circulating $\mathrm{C}$-reactive protein concentrations and risks of colon and rectal cancer: a nested case-control study within the European Prospective Investigation into Cancer and Nutrition. Am J Epidemiol, 172, 407-8.

Aleksandrova K, Boeing H, Jenab M, et al (2012) Leptin and soluble leptin receptor in risk of colorectal cancer in the european prospective investigation into cancer and nutrition cohort. Cancer Res, 72, 5328-7.

Alemán JO, Eusebi LH, Ricciardiello L, et al (2014). Mechanisms of obesity-induced gastrointestinal neoplasia. Gastroenterology, 146, 357-3.

Aloulou N, Bastuji-Garin S, Le Gouvello S, et al (2008). Involvement of the leptin receptor in the immune response in intestinal cancer. Cancer Res, 68, 9413-2.

American Cancer Society (2014). Cancer facts \& figures 2014. atlanta, ga: american cancer society. colorectal cancer detailed guide.

Apte RN, Voronov E (2002). Interleukin-1-a major pleiotropic cytokine in tumor-host interactions. Semin Cancer Biol, 12, 277-80.

Balkwill F, Charles KA, Mantovani A (2005). Smoldering and polarized inflammation in the initiation and promotion of malignant disease. Cancer Cell, 7, 211-7.

Bardou M, Barkun AN, Martel M (2013). Obesity and colorectal cancer. Gut, 62, 933-7.

Bendardaf R, Buhmeida A, Hilska M, et al (2008). VEGF-1 expression in colorectal cancer is associated with disease localization, stage, and long-term disease-specific survival. Anticancer Res, 28, 3865-70.

Birmingham JM, Busik JV, Hansen-Smith FM, Jenifer I, Fenton JI (2009). Novel mechanism for obesity-induced colon cancer progression. Carcinogenesis, 30, 690-7.

Catalán V, Gómez-Ambrosi J, Rodríguez A, et al (2011). Up-regulation of the novel proinflammatory adipokines lipocalin-2, chitinase-3 like-1 and osteopontin as well as angiogenic-related factors in visceral adipose tissue of patients with colon cancer. $J$ Nutr Biochem, 22, 634-1.

Chambers AF, Groom AC, MacDonald IC (2002). Dissemination and growth of cancer cells in metastatic sites. Nat Rev Cancer, 2, 563-2.

Choe EK, Kim D, Kim HJ, Park KJ (2013). Association of visceral obesity and early colorectal neoplasia. World $J$ Gastroenterol, 19, 8349-6.

Coelho M, Oliveira T, Fernandes R (2013). Biochemistry of adipose tissue: an endocrine organ. Arch Med Sci, 9, 191-0.

Cole BF, Logan RF, Halabi S, et al (2009). Aspirin for the chemoprevention of colorectal adenomas: metaanalysis of the randomized trials. J Natl Cancer Inst, 101, 256-6.

Endo H, Hosono K, Uchiyama T, et al (2011). Leptin acts as a growth factor for colorectal tumours at stages subsequent to tumour initiation in murine colon carcinogenesis. Gut, 60, 1363-1.

Eldesoky A, Shouma A, Mosaad Y, Elhawary A(2011). Clinical relevance of serum vascular endothelial growth factor and interleukin- 6 in patients with colorectal cancer. Saudi Journal of Gastroenterology, 17, 170-3.

Erarslan E, Turkay C, Koktener A, et al (2009). Association of visceral fat accumulation and adiponectin levels with colorectal neoplasia. Dig Dis Sci, 54, 862-8.

Erkasap N, Ozkurt M, Erkasap S, et al (2013). Leptin receptor (Ob-R) mRNA expression and serum leptin concentration in patients with colorectal and metastatic colorectal cancer. Braz J Med Biol Res, 46, 306-0.

Erreni M, Mantovani A, Allavena P (2011). Tumor-associated Macrophages (TAM) and inflammation in colorectal cancer. Cancer Microenviron, 4, 141-4.

Esposito K, Chiodini P, Coalo A, Lenzi A, Giugliano D (2012). Metabolic syndrome and risk of cancer a systematic review and meta-analysis. Diabetes Care, 35, 2402-1.

Ferlay J, Steliarova-Foucher E, Lortet-Tieulent J, et al (2013). Cancer incidence and mortality worldwide: IARC cancer base No. 11 Lyon, France: international agency for research on cancer, 2013. Eur J Cancer, 49, 1374-3.

Frezza EE, Wachtel MS, Internati MCh (2006). Influence of obesity on the risk of developing colon cancer. Gut, 55, 285-1.

Franklin RA, Liao W, Sarkar A, et al (2014). The cellular and molecular origin of tumor-associated macrophages. Science, 344, 921-5.

Geng L, Chaudhuri A, Talmon G, Wisecarver JL, Wang J (2013). TGF-beta suppresses VEGFA-mediated angiogenesis in colon cancer metastasis. PLOS ONE, 8, 59918

Gialamas SP, Sergentanis TN, Antonopoulos CN, et al (2013). Circulating leptin levels and risk of colorectal cancer and adenoma: a case-control study and meta-analysis. Cancer Causes Control, 24, 2129-1.

Gordon S (2003). Alternative activation of macrophages. Nat Rev Immunol, 3, 23-5.

Gregor MF, Hotamisligil GS (2011). Inflammatory mechanisms in obesity. Annu Rev Immunol, 29, 415-5.

Gulcelik MA, Colakoglu K, Dincer H, et al (2012). Associations between adiponectin and two different cancers: breast and colon. Asian Pac J Cancer Prev, 13, 395-8.

Haggar FA, Boushey RP (2009). Colorectal cancer epidemiology: incidence, mortality, survival, and risk factors. Clin Colon Rectal Surg, 22, 191-7.

Hardwick JC, Van Den Brink GR, Offerhaus GJ, Van Deventer SJ, Peppelenbosch MP (2001). Leptin is a growth factor for colonic epithelial cells. Gastroenterology, 121, 79-0.

Healy LA, Howard JM, Ryan AM, et al (2012). Metabolic syndrome and leptin are associated with adverse pathological features in male colorectal cancer patients. Colorectal Disease, 14, 157-5. 
Hermsen M, Postma C, Baak J, et al (2002). Colorectal adenoma to carcinoma progression follows multiple pathways of chromosomal instability. Gastroenterology, 123, 1109-9.

Ho GY, Wang T, Gunter MJ, et al (2012). Adipokines linking obesity with colorectal cancer risk in postmenopausal women. Cancer Res, 72, 3029-7.

Hoda MR, Keely SJ, Bertelsen LS, et al (2007). Leptin acts as a mitogenic and antiapoptotic factor for colonic cancer cells. Br J Surg, 94, 346-4.

Hong S, Cai Q, Chen D, et al (2012). Abdominal obesity and the risk of colorectal adenoma: a meta-analysis of observational studies. Eur J Cancer Prev, 21, 523-1.

Hotamisligil GS (2006). Inflammation and metabolic disorders. Nature, 14, 860-7.

Joshi RK, Lee SA (2014). Obesity related adipokines and colorectal cancer: a review and meta-analysis. Asian Pac J Cancer Prev, 15, 397-5.

Kabat GC, Kim MY, Peters U, et al (2012). A longitudinal study of the metabolic syndrome and risk of colorectal cancer in postmenopausal women. Eur J Cancer Prev, 21, 326-2.

Kadowaki T, Yamauchi T (2005). Adiponectin and adiponectin receptors. Endocr Rev, 26, 439-1.

Kalluri R, Weinberg RA (2009). The basics of epithelialmesenchymal transition. J Clin Invest, 119, 1420-8.

Kaneko R, Nakazaki N, Tagawa T, et al (2014). A new index of abdominal obesity which effectively predicts risk of colon tumor development infemale Japanese. Asian Pac J Cancer Prev, 15, 1005-10.

Kang HW, Kim D, Kim HJ, et al (2010). Visceral obesity and insulin resistance as risk factors for colorectal adenoma: a cross-sectional, case-control study. Am J Gastroenterol, 105, 178-7.

Kim HK, Song KS, Park YS, et al (2003). Elevated levels of circulating platelet microparticles, VEGF, IL-6 and RANTES in patients with gastric cancer: possible role of a metastasis predictor. Eur J Cancer, 39, 184-1.

Knupfer H, Preiss R (2010). Serum interleukin-6 levels in colorectal cancer patients-a summary of published results. Int J Colorectal Dis, 25, 135-40.

Landskron G, De la Fuente M, Thuwajit P, Thuwajit Ch, Hermoso MA (2014). Chronic inflammation and cytokines in the tumor microenvironment. J Immunol Res, 2014, 1-9.

Lee YS, Choi I, Ning Y, et al (2012). Interleukin-8 and its receptor CXCR2 in the tumour microenvironment promote colon cancer growth, progression and metastasis. $\mathrm{Br} \mathrm{J}$ Cancer, 106, 1833-1.

Lewis AM, Varghese S, Xu H, Alexander HR (2006). Interleukin-1 and cancer progression: the emerging role of interleukin-1 receptor antagonist as a novel therapeutic agent in cancer treatment. $J$ Transl Med, 4, 48.

Li L, Sarver AL, Khatri R, et al (2014). Sequential expression of miR-182 and miR-503 cooperatively targets FBXW7, contributing to the malignant transformation of colon adenoma to adenocarcinoma. $J$ Pathol, 234, 488-1.

Liu H, Wan D, Pan Z, et al (2011). Expression and biological significance of leptin, leptin receptor, VEGF, and CD34 in colorectal carcinoma. Cell Biochem Biophys, 60, 241-4.

Lukas M (2010). Inflammatory bowel disease as a risk factor for colorectal cancer. Dig Dis, 28, 619-4.

Mariani F, Sena P, Roncucci L (2014). Inflammatory pathways in the early steps of colorectal cancer development. World J Gastroenterol, 20, 9716-1.

Martins SF, Garcia EA, Luz MA, et al (2013). Clinicopathological correlation and prognostic significance of VEGF-A, VEGF-C, VEGFR-2 and VEGFR-3 expression in colorectal cancer. Cancer Genomics Proteomics, 10, 55-7.

Mattar MC, Lough D, Pishvaian MJ, Charabaty A (2011).
Current management of inflammatory bowel disease and colorectal cancer. Gastrointest Cancer Res, 4, 53-1.

Mariani F, Sena P, Roncucci L (2014). Inflammatory pathways in the early steps of colorectal cancer development. World J Gastroenterol, 20, 9716-1.

Morrison DS, Parr CL, Lam TH, et al (2013). Behavioural and metabolic risk factors for mortality from colon and rectum cancer: analysis ofdata from the Asia-Pacific Cohort Studies Collaboration. Asian Pac J Cancer Prev, 14, 1083-7.

Mosser DM, Edwards JP (2008). Exploring the full spectrum of macrophage activation. Nat Rev Immunol, 8, 958-6.

Nishida C, Ko GT, Kumanyika S (2010). Body fat distribution and noncommunicable diseases in populations: Overview of the 2008 WHO expert consultation on waist circumference and waist-hip ratio. Eur J Clin Nutr, 64, 2-5.

Ognunwobi OO, Bealses LP (2007). The anti-apoptotic and growth stimulatory actions of leptin in human colon cancer cells involves activation of JNK mitogen activated protein kinase, JAK2 and PI3 kinase/Akt. Int J Colorectal Dis, 22, 401-9.

Osório-Costa F, Carvalheira JBC (2013). TNF- $\alpha$ in obesityassociated colon cancer. Transl Gastrointest Cancer, 2, 179-3.

Otake S, Takeda H, Suzuki Y, et al (2005). Association of visceral fat accumulation and plasma adiponectin with colorectal adenoma: evidence for participation of insulin resistance. Clin Cancer Res, 11, 3642-6.

Paik SS, Jang SM, Jang KS, et al (2009). Leptin expression correlates with favorable clinicopathologic phenotype and better prognosis in colorectal adenocarcinoma. Ann Surg Oncol, 16, 297-3.

Pischon T, Lahmann PH, Boeing H, et. Al (2006). Body size and risk of colon and rectal cancer in the European Prospective Investigation Into Cancer and Nutrition (EPIC). J Natl Cancer Inst, 98, 920-1.

Procaccini C, De Rosa W, Galgani Carbone F, et al (2013). Role of adipokines signaling in the modulation of T cells function. Front. Immunol, 4, 332 .

Roberts DL, Dive C, Renehan AG (2010). Biological mechanisms linking obesity and cancer risk:new perspectives. Annu Rev Med, 61, 301-6.

Saely C, Geiger K, Drexel H (2012). Browns versus white adipose tissue: a mini-review. Gerontol, 58, 120-2.

Sauter ER, Garofalo C, Hewett J, et al (2004). Leptin expression in breast nipple aspirate fluidn (NAF) and serum isinfluenced by body mass index (BMI) but not by the presence of breast cancer. Horm Metab Res, 36, 336-40.

Saxena NK, Sharma D, Ding X, et al (2007). Concomitant activation of the JAK/STAT, PI3K/AKT, and ERK signaling is involved in leptin-mediated promotion of invasion and migration of hepatocellular carcinoma cells. Cancer Res, 67, 2497-7.

Schneider MR, Hoeflich A, Fischer JR, et al (2000). Interleukin-6 stimulates clonogenic growth of primary and metastatic human colon carcinoma cells. Cancer Lett, 151, 31-8.

Serhan CN (2007). Resolution phase of inflammation: novel endogenous anti-inflammatory and proresolving lipid mediators and pathways. Annu Rev Immunol, 25, 101-7.

Simms N, Rajput A, Sharratt EA, et al (2012). Transforming growth factor- $\beta$ suppresses metastasis in a subset of human colon carcinoma cells. BMC Cancer, 12, 221.

Solinas G, Germano G, Mantovani A, Allavena P (2009). Tumor-associated macrophages (TAM) as major players of the cancer-related inflammation. J Leukoc Biol, 86, 1065-3.

Stattin P, Lukanova A, Biessy C, et al (2004). Obesity and colon cancer: does leptin provide a link? Int J Cancer, 109, 149-2.

Stocks T, Lukanova A, Johansson M, et al (2008). Components 
of the metabolic syndrome and colorectal cancer risk; a prospective study. Int J Obes, 32, 304-4.

Tae ChH, Kim SE, Jung SA, et al (2014). Involvement of adiponectin in early stage of colorectal carcinogenesis. BMC Cancer, 14, 811 .

Talmadge JE, Donkor M, Scholar E (2007). Inflammatory cell infiltration of tumors: Jekyll or Hyde. Cancer Metastasis Rev, 26, 373-80.

Tamakoshi K, Toyoshima H, Wakai K, et al (2005). Leptin is associated with an increased female colorectal cancer risk: a nested case-control study in Japan. Oncol, 68, 454-1.

Tchernof A, Després JP (2013). Pathophysiology of human visceral obesity: an update. Physiol Rev, 93, 359-4.

Terzic J, Grivennikov S, Karin E, Karin M (2010). Inflammation and colon cancer. Gastroenterology, 138, 2101-4.

Tsilidis KK, Erlinger TP, Rifai N, et al (2008). C-reactive protein and colorectal adenoma in the CLUE II cohort. Cancer Causes Control, 19, 559-7.

Tsilidis KK, Branchini C, Guallar E, et al (2008). Creactive protein and colorectal cancer risk: a systematic review of prospective studies. Int J Cancer, 123, 1133-0.

Tutino V, Notarnicola M, Guerra V, Lorusso D, Caruso MG (2011). Increased soluble leptin receptor levels are associated with advanced tumor stage in colorectal cancer patients. Anticancer Res, 31, 3381-3.

Vendramini-Costa DB, Carvalho JE (2012). Molecular link mechanisms between inflammation and cancer. Curr Pharm Des, 18, 3831-2.

Voronov E, Shouval DS, Krelin Y, et al (2003). IL-1 is required for tumor invasiveness and angiogenesis. Proc Natl Acad Sci USA, 100, 2645-50.

Wei EK, Giovannucci E, Fuchs ChS, Willett WC, Mantzoros $\mathrm{ChS}$ (2005). Low plasma adiponectin levels and risk of colorectal cancer in men: a prospective study.J Natl Cancer Inst, 97, 1688-4.

Winkels RM, Heine Broring RC, van Zutphen M, et al (2014). The COLON study: Colorectal cancer: Longitudinal, observational study on nutritional and lifestyle factors that may influence colorectal tumour recurrence, survival and quality of life. BMC Cancer, 14, 374.

Wiwanitkit V (2007). Interaction between leptin and leptin receptor in gastric carcinoma: gene ontology analysis. Rev Esp Enferm Dig, 99, 201-5.

Wu J, Cai Q, et. al. (2013). Circulating C-reactive protein and colorectal cancer risk: a report from the Shanghai Men's Health Study. Carcinogenesis, 34, 2799-3.

Yoneda K, Tomimoto A, Endo H, et al (2008). Expression of adiponectin receptors, AdipoR1 and AdipoR2, in normal colon epithelium and colon cancer tissue. Oncol Rep, 20, 479-3. 